\title{
The relationship between chondromalacia patella, medial meniscal tear and medial periarticular bursitis in patients with osteoarthritis
}

\author{
Mustafa Resorluํ, Davut Doner², Ozan Karatag', Canan Akgun Toprak ${ }^{1}$ \\ ${ }^{1}$ Department of Radiology, Canakkale Onsekiz Mart University, Faculty of Medicine, Canakkale, Turkey \\ 2 Department of Physical Medicine and Rehabilitation, Canakkale Onsekiz Mart University, Faculty of Medicine, \\ Canakkale, Turkey
}

Radiol Oncol 2017; 51(4): 401-406.

Received 9 May 2017

Accepted 26 September 2017

Correspondence to: Mustafa Resorlu, M.D., Canakkale Onsekiz Mart University, Terzioğlu Yerleșkesi, Barbaros Mh, 17100, Canakkale, Turkey. Phone: +90 505454 8722; Fax: +90 286 2180393; E-mail: mustafaresorlu77@gmail.com

Disclosure: No potential conflicts of interest were disclosed.

Background. This study investigated the presence of bursitis in the medial compartment of the knee (pes anserine, semimembranosus-tibial collateral ligament, and medial collateral ligament bursa) in osteoarthritis, chondromalacia patella and medial meniscal tears.

Patients and methods. Radiological findings of 100 patients undergoing magnetic resonance imaging with a preliminary diagnosis of knee pain were retrospectively evaluated by two radiologists. The first radiologist assessed all patients in terms of osteoarthritis, chondromalacia patella and medial meniscal tear. The second radiologist was blinded to these results and assessed the presence of bursitis in all patients.

Results. Mild osteoarthritis (grade I and II) was determined in 55 patients and severe osteoarthritis (grade III and IV) in 45 cases. At retropatellar cartilage evaluation, 25 patients were assessed as normal, while 29 patients were diagnosed with mild chondromalacia patella (grade I and II) and 46 with severe chondromalacia patella (grade III and IV). Medial meniscus tear was determined in 51 patients. Severe osteoarthritis and chondromalacia patella were positively correlated with meniscal tear ( $p<0.001$ and $p=0.018$, respectively). Significant correlation was observed between medial meniscal tear and bursitis in the medial compartment $(p=0.038)$. Presence of medial periarticular bursitis was positively correlated with severity of osteoarthritis but exhibited no correlation with chondromalacia patella $(p=0.023$ and $p=0.479$, respectively). Evaluation of lateral compartment bursae revealed lateral collateral ligament bursitis in 2 patients and iliotibial bursitis in 5 patients.

Conclusions. We observed a greater prevalence of bursitis in the medial compartment of the knee in patients with severe osteoarthritis and medial meniscus tear.

Key words: medial periarticular bursitis; medial meniscal tear; osteoarthritis

\section{Introduction}

Although osteoarthritis (OA), a common joint disease, is not fatal it compromises quality of life. It has become more important in terms of public health with the increase in the average life span associated with advances in the medical sphere. The process begins with progressive loss of joint cartilage and gradually causes meniscal, muscle, bone tissue, ligament and bursa pathologies. ${ }^{1}$ Various factors are implicated in the link between aging and OA. The most important of these are decreased cartilage perfusion, impaired joint morphology, increased ligament laxity, decreased anabolic response and 
thinning in the cartilage plate. ${ }^{2}$ Female gender and obesity are other important risk factors in addition to aging. Hormonal changes in particular occurring in women in the postmenopausal period are also a significant predisposing factor. ${ }^{3}$ Obesity also leads to osteoarthritis, not only through its mechanical effect but also due to its metabolic consequences, and has an adverse effect on clinical findings. ${ }^{1,3}$ Since it results in a mechanical disadvantage for lower extremity functions, pain is more frequent and more severe. ${ }^{4}$ Another clinical condition frequently encountered together with aging is chondromalacia patella (CP). Vascular insufficiency, patellar variations, trauma, dislocation, fracture, rheumatological diseases and impairment of mechanical stress balance on the joint are implicated in the etiology. ${ }^{5}$ The principal changes in cartilage tissue are softening, swelling, fissuring or ulceration. ${ }^{5}$ There are several superficially or deeply located bursae in the knee joint, their principal function being to reduce friction between the surfaces. Pes anserine (PA) bursa, semimembranosus-tibial collateral ligament (SM-TCL) bursa and medial collateral ligament (MCL) bursa inflammations may cause to medial knee pain. Similarly, inflammation in the iliotibial or lateral collateral ligament (LCL) bursae in the lateral of knee also lead to knee pain. Etiological factors such as recurring trauma, impaired joint stability and joint overloading play a role in the development of bursitis. ${ }^{6}$ Magnetic resonance imaging (MRI) is the gold standard radiological technique in the assessment of pathologies of the ligament, meniscus, cartilage, bursa and bone marrow in the knee joint.7 This study investigated the relationship between pathologies, such as meniscal tear, $\mathrm{OA}$ and $\mathrm{CP}$, affecting knee mechanics and bursitis in medial compartment of knee

\section{Patients and methods}

\section{Patients}

MR images from 100 patients with OA of the knee were evaluated retrospectively. The patients included in our study group were selected from among individuals presenting to our hospital in 2014-2016. Individuals aged 65 or over were included in the study group, irrespective of gender. Patient selection was based on the order of patients on the work station. Patients with OA determined with conventional radiography were included. Exclusion criteria included presence of acute trauma or fracture, inadequate image quality, rheumatological disease or history of knee surgery.

\section{Imaging protocol}

All patients were evaluated with a routine knee imaging protocol on a 1.5 Tesla MRI unit (Signa Excite; GE Medical Systems, Wisconsin, USA). Section thicknesses were $3.5 \mathrm{~mm}$ in sagittal images and $4 \mathrm{~mm}$ in axial and coronal images. The fluid-sensitive sequences were performed with fat suppression. The imaging protocol consisted of a sagittal T1 weighted spin-echo sequence (540 $\mathrm{ms} / 11.3 \mathrm{~ms}$, repetetion time [TR]/echo time [TE], 320x224 matrix, number of excitations [NEX] 1.0), sagittal T2 weighted spin-echo (4480 ms/85 ms, TR/ TE; 256x256 matrix; NEX,1.0) axial proton density (3300 ms/34.7 ms, [TR]/ [TE]; [NEX], 1.0; 320x224 matrix), sagittal proton density sequence (4480 ms/45 ms, TR/TE; NEX,1.0; 320x224 matrix) and a coronal proton density sequence $(3620 \mathrm{~ms} / 31.8 \mathrm{~ms}$, TR/TE; 320x224 matrix; NEX,1.0).

\section{Image assessment}

The radiological findings of 100 patients with OA of the knee undergoing MRI with a preliminary diagnosis of knee pain were retrospectively evaluated by two radiologists. The first radiologist assessed all patients in terms of $\mathrm{OA}, \mathrm{CP}$, medial meniscal damage and anterior and posterior cruciate ligament pathologies. The second radiologist, blinded to the results, investigated the presence of bursitis in all patients. An approval was obtained from Canakkale Onsekiz Mart University Ethics Committee and written informed consent was waived because of the retrospective nature of the study. Presence of osteophytes, narrowing in the articular space and sclerotic changes were assessed with radiography. OA grading was based on radiography and the Kellgren-Lawrence system grading scale. ${ }^{8}$ Grades I and II were regarded as mild and grades III and IV as severe.

The Noyes Chondral Injury Classification system was used to classify CP. Grade 0 was regarded as normal, grade I as focal signal increase in cartilage, grade II as fissure or ulcer in cartilage of up to $50 \%$, grade III as greater than $50 \%$ loss in cartilage thickness and grade IV as full thickness cartilage defect and subchondral edema. Grade I and II were regarded as mild and grade III and IV as severe. Meniscal damage was classified under three groups - Group 1; normal, Group 2; degenerative signal increase without tear, and Group 3; tear opening onto at least one articular surface. Enlargement in the bursa, fluid deposition, wall thickening and accompanying edema in close proximity were in- 
terpreted in favor of bursitis. ${ }^{9}$ Hypointense signal changes on T1W images and hyperintense signal changes on TW2 images reflecting increased concentration of compounds containing $\mathrm{H}^{+}$ions in the bone medulla were interpreted in favor of bone marrow edema. ${ }^{10}$

\section{Statistical analysis}

Statistical analysis was performed on SPSS 20.0 (SPSS Inc., Chicago, IL, USA) software. The Kolmogorov-Smirnov/Shapiro-Wilk tests were used to assess normal distribution of variables. Descriptive data were expressed as mean, standard deviation and minimum and maximum values, frequency and percentage values. The $t$ test was used to compare means in independent groups and the chi square test in the analysis of categoric variables. Relations between constant variables were investigated using Kendall's correlation analysis. $\mathrm{P}<0.05$ was regarded as statistically significant.

\section{Results}

Gender distribution was 54 female (54\%) and 46 male $(46 \%)$, and the mean age was $70.47 \pm 5.35$ years (min 65, max 84). Mild OA (grade I and II) was determined in 55 patients and severe OA (grade III and grade IV) in 45 . There was no difference between male and female patients in terms of severity of OA $(p=0.170)$. At evaluation of the retropatellar cartilage, 25 patients were normal, while mild CP (grade I and II) was determined in 25 and severe CP (grade III-IV) in 46.

Since lateral meniscal tear was determined in only six patients, this could not be subjected to statistical analysis. At radiological examination of the medial meniscus, 4 patients were normal, while degenerative signal increase was present in 45 patients and meniscal tear in 51. Meniscal tear was present in 36 of the 45 patients with severe OA and in 15 of the 55 patients with mild OA, and the correlation between severity of $\mathrm{OA}$ and meniscal tear was statistically significant $(p<0.001)$. Similarly, significant correlation was determined between $\mathrm{CP}$ and meniscal tear $(p=0.018)$. Bursitis in the medial compartment was present in 26 of the 51 patients with medial meniscal tear, and in 15 of the 49 patients with no tear $(p=0.038)$. Bone marrow edema in the tibia or femur was determined in 45 patients. Presence of bone marrow edema was positively correlated with severity of OA, but not with CP (p $<0.001$ and $p=0.93$, respectively). Patients' demo-
TABLE 1. Patients' demographic characteristics and radiological findings

\begin{tabular}{lc}
\hline Mean age, years & $70.47 \pm 5.5$ \\
\hline Gender, no. & 54 \\
Female & 46 \\
Male & \\
Osteoarthritis, $\mathrm{n}$ & 55 \\
Mild (Grade I and II) & 45 \\
Severe (Grade III and IV) & \\
Chondromalacia patella, n & 25 \\
Normal & 29 \\
Mild (Grade I and II) & 46 \\
Severe (Grade III and IV) & \\
Medial meniscal tear, no & 4 \\
Normal & 45 \\
Degeneration & 51 \\
Tear & 45 \\
Bone marrow edema, no & \\
Others & 4 \\
Enchondroma & 4 \\
Synovial chondromatosis & \\
Osteochondroma & \\
\hline
\end{tabular}

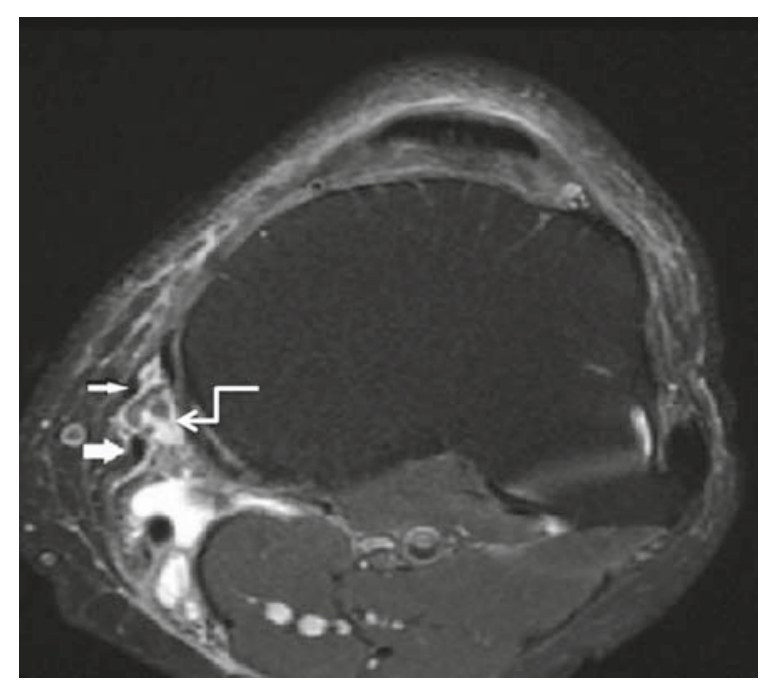

FIGURE 1. A 70 year-old patient presented with knee pain. Axial MR image shows sartorius (thin arrow) and gracilis tendon (thick arrow) in the patient with pes anserine bursitis (curved arrow).

graphic characteristics and radiological findings are shown in Table 1.

Bursitis was determined in at least one of the medial periarticular bursae in 41 patients, the most common form being PA bursitis (Figure 1). Bursitis cases determined at MRI are shown in Table 2. 

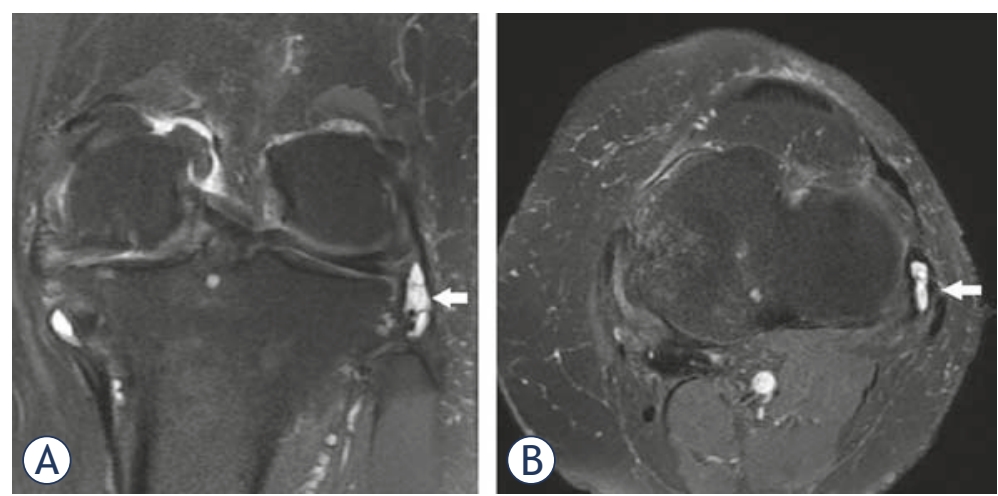

FIGURE 2. Coronal (A) and axial (B) proton-density fat-suppressed images of knee show LCL bursitis in a 70 years old patient.
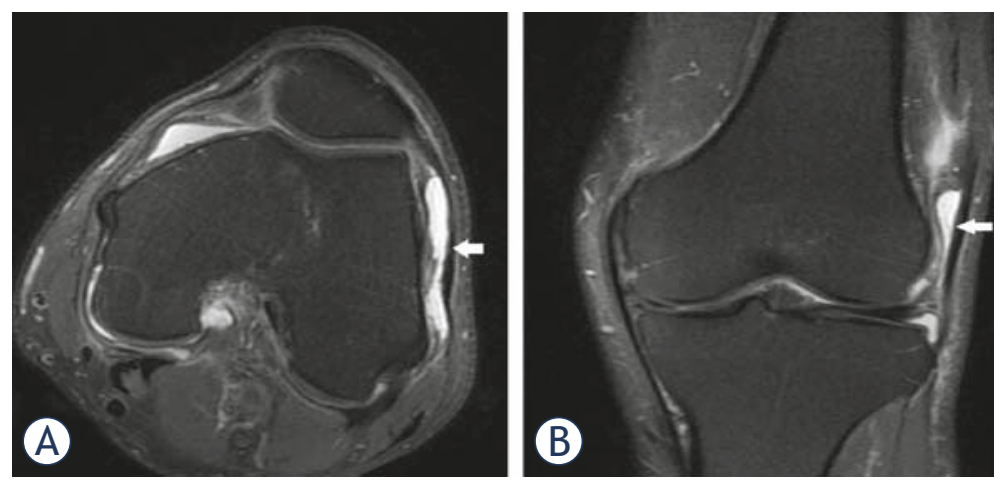

FIGURE 3. A 80 year-old patient presented with pain and tenderness in knee joint Axial (A) and coronal (B) images demonstrate a fluid collection in the dilated iliotibial bursa. partment is shown in Table 3. Baker's cyst was determined in 36 patients, the prevalence increasing with severity of $\mathrm{OA}$ but was not correlated with $\mathrm{CP}$ $(\mathrm{p}=0.044$ and $\mathrm{p}=0.264$, respectively).

Positive correlation was present between severity of $\mathrm{OA}$ and $\mathrm{CP}(\mathrm{p}=0.03)$. Evaluation of lateral compartment bursae revealed lateral collateral bursitis in two patients and iliotibial bursitis in five (Figure 2 and 3). Prepatellar bursitis in the anterior compartment was determined in four patients, infrapatellar bursitis in four and suprapatellar bursitis in three. Additionally, synovial enchondromatosis was present in four patients, enchondroma in four and osteochondroma in one.

\section{Discussion}

The knee joint is frequently subjected to trauma, and degenerative changes occur with aging. Trauma, degenerative changes, morphological variations, mechanical stress impairments and aging are factors involved in the etiology of $\mathrm{OA}, \mathrm{CP}$, bursitis and medial meniscal tear. ${ }^{3,11}$ Common risk factors are one of the reasons why pathologies in the knee joint affect more than one anatomical structure, rather than remaining local. Moreover, pathologies that begin locally induce morphological and functional changes in other anatomical structures by compromising biomechanics. ${ }^{11,12}$ Burger et al. investigated the development of OA as a result of inadequate meniscal tear repair in an animal study. ${ }^{12}$ They reported that OA developed in the medial tibiofemoral and patellofemoral joint due to insufficient meniscal tear repair. Cicuttini et al. showed gradual loss of cartilage in patients under-

TABLE 3. The relation between osteoarthritis, chondromalacia patella, meniscal tear and medial periarticular bursitis

\begin{tabular}{lccc}
\hline & \multicolumn{4}{c}{ Medial compartment bursitis } \\
\cline { 2 - 4 } & Yes & No & $p$ \\
\hline Osteoarthritis & 17 & 38 & 0.023 \\
Mild $(n=55)$ & 24 & 21 & \\
Severe $(n=45)$ & & & \\
Chondromalacia Patella & & 17 & \\
Normal $(n=25)$ & 14 & 15 & 0.479 \\
Mild $(n=29)$ & 19 & 27 & \\
Severe $(n=46)$ & 15 & 34 & 0.038 \\
Meniscal Tear & & \multicolumn{3}{c}{25} \\
No $(n=49)$ & 26 & \multicolumn{3}{c}{} \\
Yes $(n=51)$ & & &
\end{tabular}


going partial meniscectomy ${ }^{13}$ The significant correlation observed in our study between both $\mathrm{OA}$ and CP with meniscal tear $(p<0.001$ and $p=0.018)$ was compatible with these findings. Additionally, the correlation between severity of OA and $\mathrm{CP}(\mathrm{p}$ $=0.030$ ) also supports the previous literature..$^{12,14}$ These findings all suggest that these pathologies induce one another as a result of changes in knee biomechanics and morphology. Furthermore, common risk factors such as advanced age and degeneration reinforce this association.

Inflammation occurring in the PA, SM-TCL (Figure 4) and MCL bursa in the medial of knee is an important cause of pain. Obesity, degenerative joint disease, valgus deformity, trauma, impaired joint stability, excessive loading on the joint and sporting activity are important causes of inflammation in the bursae. ${ }^{15}$ Some authors have suggested that the structure of the female pelvis creates a predisposition to bursitis. ${ }^{16}$ We determined no relation between gender and the prevalence of bursitis $(\mathrm{p}=0.954)$. Toktas et al. investigated the prevalence of pes anserine bursitis in patients with OA. ${ }^{17}$ They determined a greater pes anserine thickness not only in OA patients with bursitis, but also in OA patients without bursitis compared to the control group. We selected all our cases from OA patients and took the OA grade (mild and severe) as our reference points. We also investigated the presence of SM-TCL and MCL bursitis in addition to PA bursitis. We observed higher prevalence of medial periarticular bursitis in patients with severe OA compared to those with mild OA $(p=0.023)$. Our results are compatible with recent studies revealing as association between $\mathrm{OA}$ and pes anserine bursitis. ${ }^{16}$

Another important finding of this study was a positive correlation between medial meniscal tear and medial compartment bursitis. Bursitis was present in 26 of the 51 patients in whom medial meniscal tear was determined, and in 15 of the 49 patients with no tear. The difference was statistically significant $(p=0.038)$. Various factors affect the positive relation between prevalence of bursitis and severity of OA and medial meniscal tear. The first are similar etiological factors. Second are structural changes associated with OA and meniscal tear and impairment of joint stability. ${ }^{16}$ Despite the common etiological factors and the effect on joint biomechanics, we determined no relation between $\mathrm{CP}$ and medial compartment bursitis. We think that this finding was affected by patellar and trochlear morphology. Variations such as patella alta and baja influence the development of

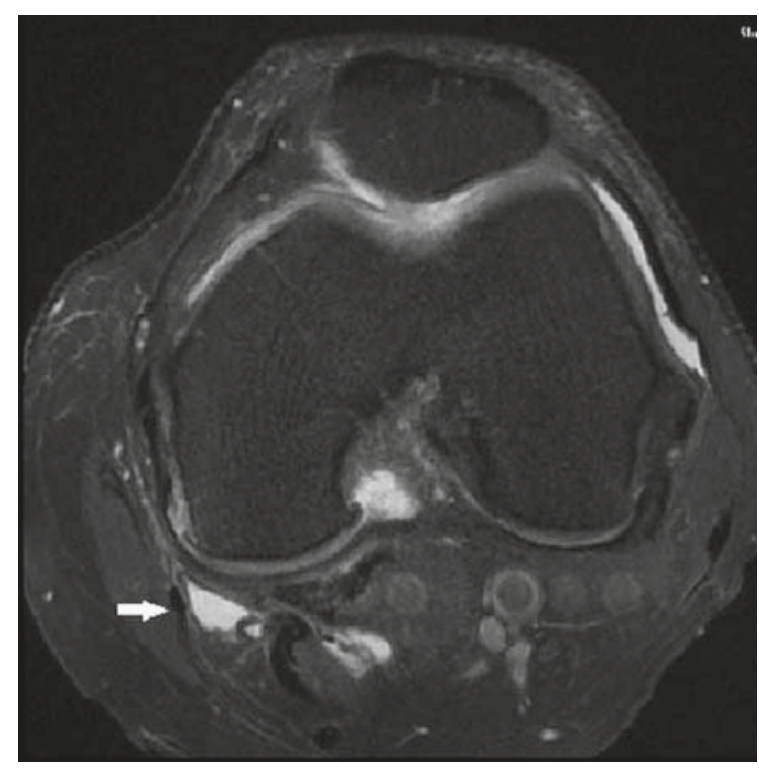

FIGURE 4. The axial fat saturated proton density weighted image demonstrates a fluid collection in the SM-TCL bursa.

CP. Additionally, it has been suggested that a short medial facet increases mechanical stress on cartilage and causes CP. 5,18 One recent study proposed the sulcus angle/trochlear depth ratio as a powerful predictor. ${ }^{11}$ One limitation of our study is that patellar and trochlear morphology were not evaluated. Another limitation is that due to its retrospective nature patients were not assessed in terms of diabetes during the study.

The prevalence of Baker's cyst was 36\%, iliotibial bursitis $5 \%$ and LCL bursitis $2 \%$. In their study of 163 patients, Hayashi et al. reported prevalences of popliteal cyst of $40 \%$ and of iliotibial bursitis of $1 \%{ }^{19}$ Baker's cyst was the most common lesion in both studies, and the results were compatible. A relation was determined between Baker's cyst and severity of OA in our study, but there was no correlation between bursitis in the medial compartment and Baker's cyst $(\mathrm{p}=0.044$ and $\mathrm{p}=0.954$, respectively). The positive correlation between Baker's cyst and OA is compatible with the previous literature. ${ }^{20}$ Increased joint effusion and intraarticular pressure in OA may be involved in this relationship. We observed prepatellar (4\%), infrapatellar (4\%) and suprapatellar bursitis (3\%) in the anterior of joint. Hayashi et al. reported the prevalence of prepatellar and infrapatellar bursitis at $2 \%$. The mean age of their study population was $52 \pm 6$ years, and patients without OA constituted $32 \%$ of their cases. ${ }^{19}$ The higher mean age in our study and all our patients having OA may explain the difference in the findings. 
In conclusion, we observed a correlation between the prevalence of bursitis in the medial compartment and the severity of OA and medial meniscal tear. We determined no relation between severity of $\mathrm{CP}$ and bursitis.

\section{References}

1. Ayral X, Pickering EH, Woodworth TG, Mackillop N, Dougados M. Synovitis: A potential predictive factor of structural progression of medial tibiofemoral knee osteoarthritis e results of a 1 year longitudinal arthroscopic study in 422 patients. Osteoarthr Cartil 2005; 13: 361-7. doi: 10.1016/j. joca.2005.01.005

2. Arden N, Nevitt MC. Osteoarthritis: epidemiology. Best Pract Res Clin Rheumatol 2006; 20: 3-25. doi: 10.1016/j.berh.2005.09.007

3. Creamer $P$, Lethbridge-Cejku $M$, Hochberg MC. Factors associated with functional impairment in symptomatic knee osteoarthritis. Rheumatology (Oxford) 2000; 39: 490-6.

4. de Miguel Mendieta E, Cobo Ibáñez T, Usón Jaeger J, Bonilla Hernan G, Martın Mola E. Clinical and ultrasonographic findings related to knee pain in osteoarthritis. Osteoarthritis Cartilage 2006; 14: 540. doi: 10.1016/j. joca.2005.12.012

5. Tanamas SK, Teichtahl AJ, Wluka AE, Wang Y, Davies-Tuck M, Urquhart $\mathrm{DM}$, et al. The associations between indices of patellofemoral geometry and knee pain and patella cartilage volume: a crosssectional study. BMC Musculoskelet Disord 2010; 11: 87. doi: 10.1186/1471-2474-11-87

6. Alvarez-Nemegyei J. Risk factors for pes anserinus tendinitis/bursitis syndrome: a case control study. J Clin Rheumatol 2007; 13: 63-5. doi: 10.1097/01.rhu.0000262082.84624.37

7. Nenezic $D$, Kocijancic I. The value of the sagittal-oblique MRI technique for injuries of the anterior cruciate ligament in the knee. Radiol Oncol 2013; 47 19-25. doi: 10.2478/raon-2013-0006

8. Kellgren JH, Lawrence JS. Radiological assessment of osteoarthritis. Ann Rheum Dis 1957; 16: 499-502.

9. Hirji Z, Hunjun JS, Choudur HN. Imaging of the bursae. J Clin Imaging SC 2011; 1: 22. doi: 10.4103/2156-7514.80374

10. Oztekin O, Calli C, Kitis O, Adibelli ZH, Eren CS, Apaydin M, et al. Reliability of diffusion weighted MR imaging in differentiating degenerative and infectious end plate changes. Radiol Oncol 2010; 44: 97-102. doi: 10.2478/ v10019-010-0006-z

11. Resorlu H, Zateri C, Nusran G, Goksel F, Aylanc N. The relation between chondromalacia patella and meniscal tear and the sulcus angle/trochlear depth ratio as a powerful predictor. J Back Musculoskelet Rehabil 2017; 30: 603-8. doi: 10.3233/BMR-160536.

12. Burger C, Kabir K, Mueller M, Rangger C, Mınor T, Tolba RH. Reropatellar chondromalacia associated with medial osteoarthritis after meniscus injury. One year of observations in sheep. Eur Surg Res 2006; 38: 102-8. doi: 10.1159/000093281

13. Cicuttini FM, Forbes A, Yuanyuan W, Rush G, Stuckey SL. Rate of knee cartilage loss after partial meniscectomy. J Rheumatol 2002; 29: 1954-6.

14. Kalichman L, Zhang Y, Niu J, Goggins J, Gale D, Felson DT, et al. The association between patellar alignment and patellofemoral joint osteoarthritis features-an MRI study. Rheumatology (Oxford) 2007; 46: 1303-8. doi: 10.1093/rheumatology/kem095

15. Saggini R, Di Stefano A, Dodaj I, Scarcello L, Bellomo RG. Pes anserine bursitis in symptomatic osteoarthritis patiens: A mesotehrapy Treatment study. J Altern Complement Med 2015; 21: 480-4. doi: 10.1089/acm.2015.0007

16. Helfenstein M Jr, Kuromoto J. Anserine syndrome. Rev Bras Reumatol 2010 50: 313-27. doi: 10.1590/S0482-50042010000300011

17. Toktas H, Dundar U, Adar S, Solak O, Ulaslı AM. Ultrasonographic assessment of pes anserinus tendon and pes anseinus tendinitis bursitis syndrome in patients with knee osteoarthritis. Mod Rheumatol 2015; 25: 128-33. doi: 10.3109/14397595.2014.931909
18. Endo Y, Schweitzer ME, Bordalo-Rodrigues M, Rokito AS, Babb JS. MRI quantiative morphologic analysis of patellofemoral region: lack of correlation with chondromalacia patellaet surgery. AJR Am J Roentgenol 2007; 189: 1165-8. doi: 10.2214/AJR.07.2236

19. Hayashi D, Roemer FW, Dhina Z, Kwoh CK, Hannon MJ, Moore C, et al. Longitudinal assessment of cyst-like lesions of the knee and their relation to radiographic osteoartjritis and MRI-detected effusion and synovitis in patients with knee pain. Arthitis Res Ther 2010; 12: R172. doi: 10.1186/ar3132

20. Hill CL, Gale DG, Chaisson CE, Skinner K, Kazis L, Gale ME, et al. Knee effusions, popliteal cysts, and synovial thickening: association with knee pain in osteoarthritis. J Rheumatol 2001; 28: 1330-7. 